\title{
Masking healthcare workers (HCW) and visitors was effective to reduce nosocomial (NOSO) influenza (I) during 2014/15 epidemic with reduced vaccine effectiveness
}

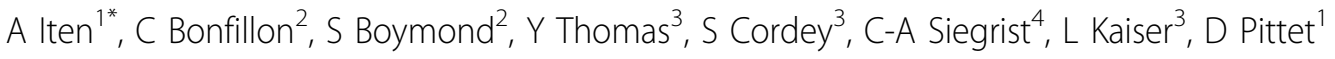 \\ From 3rd International Conference on Prevention and Infection Control (ICPIC 2015) \\ Geneva, Switzerland. 16-19 June 2015
}

\section{Introduction}

Vaccination of HCW against seasonal influenza (SI) is the cornerstone for the prevention of NOSO I. In countries where vaccination cannot be made mandatory by law, an alternative exists: HCWs' obligation to be vaccinated (VAC) or to wear a mask during SI epidemics. This is the strategy (called "Zoning") adopted by HUG since 2009. In Switzerland, in winters 2013/14 \& 2014/15, SI had similar epidemic curves but vaccine effectiveness differed. In 2014/ 15 , the trivalent vaccine did not cover the major circulating SI H3N2 strain.

\section{Objectives}

We describe 2013/14 and 2014/15 SI epidemics and NOSO at HUG.

\section{Methods}

Suspected cases of SI (respiratory symptoms, fever with chills, muscular pain, or prostration) were screened using nasopharyngeal samples analyzed by RT-PCR. Cases were defined as NOSO when symptoms occurred $>72 \mathrm{~h}$ after admission. Regular audits were performed to assess compliance with recommendations.

\section{Results}

In winter 2013/14, 309 patients were positive for I, 147 of which (47.6\%) were NOSO. Droplet precautions with single room isolation whenever possible were implemented for 261 patients $(84.5 \%)$. Of $4459 \mathrm{HCW}$ observed, $78.5 \%$ were VAC or wore a mask. In winter 2014/15, “Zoning” was

'Infection Control Program, University of Geneva Hospitals, Geneva, Switzerland

Full list of author information is available at the end of the article implemented on 31/12/2014. Early Jan 2015, a large number of SI was documented with a high proportion of NOSO: 49.2\% (92/187), in particular in internal medicine (19/28; 67.8\%). At time of audit, 992/1262 (78.6\%) HCW were VAC or wore a mask. Additional measures were implemented from $15^{\text {th }}$ January to $20^{\text {th }}$ March 2015: mandatory mask for HCW (even for VAC HCW) and visitors. Following this additional measure, 68/175 (38.8\%) cases were NOSO at HUG, in particular 19/121 (15.7\%) in internal medicine. Recommendations were followed by 2143 / 2769 (77.4\%) HCWs and 430/685 (62.8\%) visitors. Droplet precautions were implemented for $432 / 468$ (92.3\%) SI patients.

\section{Conclusion}

During the large 2014/15 epidemic with reduced SI vaccine effectiveness, mandatory mask wear for $\mathrm{HCW}$ and visitors was an effective measure to reduce NOSO I.

\section{Disclosure of interest}

None declared.

\begin{abstract}
Authors' details
'Infection Control Program, University of Geneva Hospitals, Geneva, Switzerland. ${ }^{2}$ Hospital Health Service, University of Geneva Hospitals, Geneva, Switzerland. ${ }^{3}$ Laboratory of Virology, University of Geneva Hospitals, Geneva, Switzerland. ${ }^{4}$ Department of Child and Adolescent Medicine, University of Geneva Hospitals, Geneva, Switzerland.
\end{abstract}

Published: 16 June 2015

doi:10.1186/2047-2994-4-S1-061

Cite this article as: Iten et al: Masking healthcare workers $(\mathrm{HCW})$ and visitors was effective to reduce nosocomial (NOSO) influenza (I) during 2014/15 epidemic with reduced vaccine effectiveness. Antimicrobial Resistance and Infection Control 2015 4(Suppl 1):O61. 